\title{
Machine-learning algorithm quantifies gender bias in astronomy
}

Calculation suggests papers with women first-authors have citation rates pushed down by $10 \%$.

Inga Vesper

04 November 2016

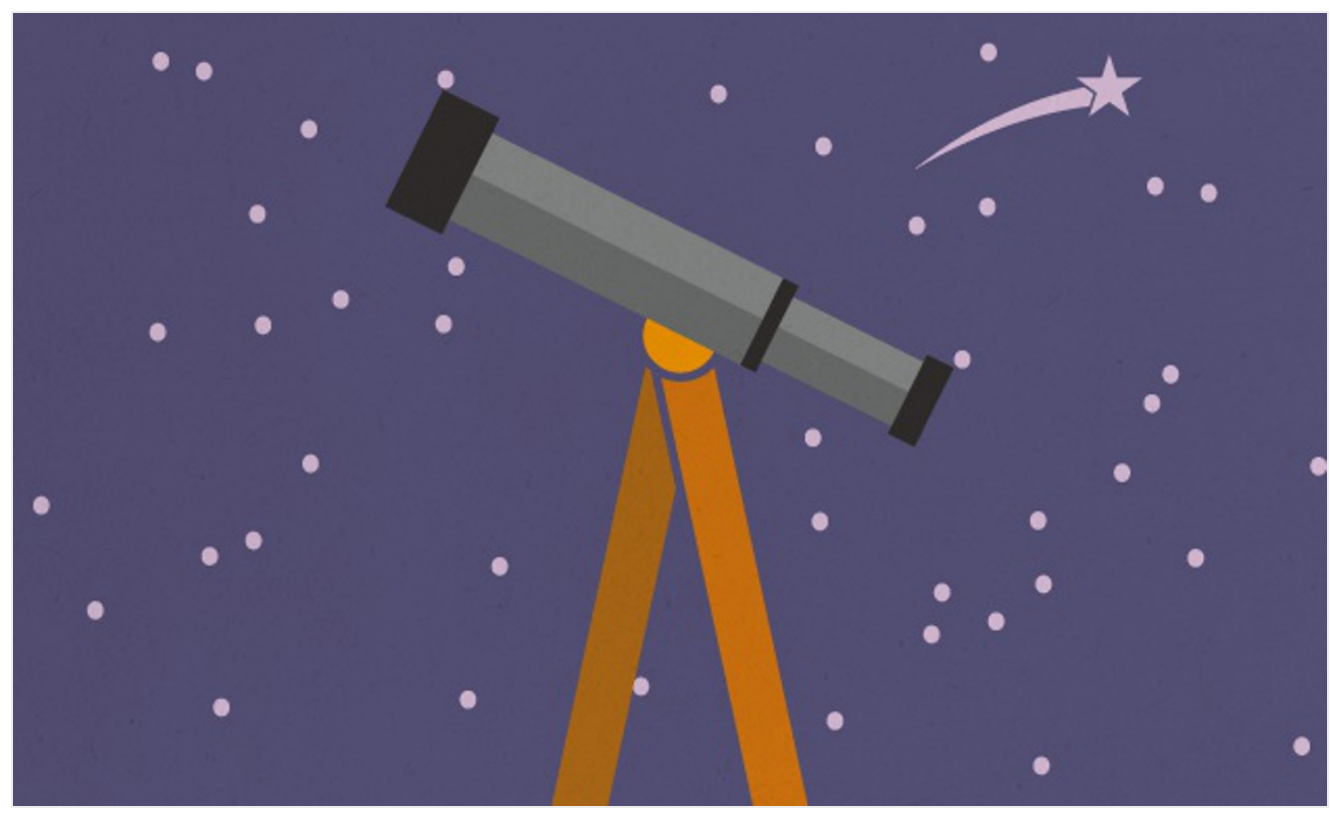

Citation rates in astronomy are stacked against women, a study that uses machine learning to quantify bias has found.

Researchers from the Swiss Federal Institute of Technology in Zurich, Switzerland, estimate that, as a result of gender bias, papers whose first authors are women receive around $10 \%$ fewer citations than do those that are first-authored by men.

Gender disparities in citation patterns have been documented across science before. But researchers have not previously tried to quantify how much of the differences are the result of gender bias. For instance, men and women may publish different types of papers; women may work in different scientific fields, and may hold less-senior positions.

But the new paper, which has not yet been peer-reviewed and was posted on the arXiv preprint server on 27 October $^{1}$, tries to account and correct for these factors. The authors declined to comment on the paper, because they hope to submit it to Nature Astronomy. But other specialists say that the analysis seems solid.

"The novelty of this paper is in dispelling the myth that gender disparity in citation can be attributed to 
specifics of the paper, rather than to gender," says Cassidy Sugimoto, an informaticist at Indiana University Bloomington. Sugimoto has also published work on gender bias in science publications ${ }^{2}$, and says that the paper's findings are "at once both terrible and terrific".

\section{Estimating gender bias}

For their study, the researchers analysed 200,000 papers in 5 journals from 1950 to 2015 . First, they

trained a machine-learning algorithm to accurately calculate the citations for each paper first-authored by a man using as many non-genderrelated factors as possible — such as the journal, field and year in which the paper was published, where the first author was located and for how many years that author had been publishing.

Then they unleashed their algorithm on the papers with female first authors. This set of papers (from 1985 onwards) had actually received around $6 \%$ fewer citations than their male-authored counterparts. But the algorithm predicted that the papers should have got $4 \%$ more citations than did those authored by men.

The authors say that the result is their "best effort" to measure gender bias, but that their results should be taken with care, because other factors might need to be weighed into their algorithm.

"This means women and men of equal quality will have unequal records," says Meg Urry, director of Yale Center for Astronomy and Astrophysics in New Haven, Connecticut, who gave advice to the Swiss researchers as they conducted their study. She adds that being cited less is likely to result in fewer grants, invitations to talks and recommendation letters. "Given how heavily our hiring process depends on these metrics, it's not surprising that women have not reached equity in academia," she says.

\section{Suppressed citations}

A good track record of citations is essential for research career progression, so the findings could go some way towards explaining the dearth of women in senior academic positions, says Karen Masters, an astronomer at the University of Portsmouth, UK.

"I have had people tell me that they won't shortlist for permanent faculty jobs unless the candidates have 100 citations on a first-author paper," she says. "So, I think it's really in getting on shortlists for jobs that this suppression in citation rate for women is going to be hurting them."

The new study also notes that women publish 19\% fewer articles than men in the 7 years after their first published paper. Astrophysicist Anna Scaife, who heads the Interferometry Centre of Excellence at Jodrell Bank observatory in the United Kingdom, says that this factor could be even more damaging than the low citation rates. "The 4-6 years following PhDs are crucial for producing the output that contributes to their first application for a permanent position," she says.

To address the problem, Masters suggests a solution straight from the astronomer's toolbox. The number of citations that women receive, she says, could be multiplied by 1.1 to eradicate the intrinsic bias. "We often correct systematic biases like that empirically in trends we view in astronomy," she says. "So I think this could be treated similarly."

Nature I doi:10.1038/nature.2016.20932

\section{- References}

1. Caplar, N., Tacchella, S. \& Birrer, S. Preprint at http://arxiv.org/abs/1610.08984 (2016).

2. Lariviere, V. et al, Nature 504, 211-213 (2013).

\section{SPRINGER NATURE}

